\title{
The cep quorum-sensing system of Burkholderia cepacia H111 controls biofilm formation and swarming motility
}

\author{
Birgit Huber, ${ }^{1}$ Kathrin Riedel, ${ }^{1}$ Morten Hentzer, ${ }^{2}$ Arne Heydorn, ${ }^{2}$ \\ Astrid Gotschlich, ${ }^{1}$ Michael Givskov, ${ }^{2}$ Søren Molin ${ }^{2}$ and Leo Eberl ${ }^{1}$
}

Author for correspondence: Leo Eberl. Tel: +49 8161 715446. Fax: +49 8161715475. e-mail: EBERL@mikro.biologie.tu-muenchen.de

1 Lehrstuhl für Mikrobiologie, Technische Universität München, Am Hochanger 4 85350 Freising, Germany

2 Department of Microbiology, DTU, Building 301, 2800 Lyngby, Denmark
Burkholderia cepacia and Pseudomonas aeruginosa often co-exist as mixed biofilms in the lungs of patients suffering from cystic fibrosis (CF). Here, the isolation of random mini-Tn5 insertion mutants of $B$. cepacia $\mathrm{H} 111$ defective in biofilm formation on an abiotic surface is reported. It is demonstrated that one of these mutants no longer produces $\mathbf{N}$-acylhomoserine lactones (AHLs) due to an inactivation of the cepR gene. cepR and the cepl AHL synthase gene together constitute the cep quorum-sensing system of $B$. cepacia. By using a gene replacement method, two defined mutants, H111-I and H111-R, were constructed in which cepl and cepR, respectively, had been inactivated. These mutants were used to demonstrate that biofilm formation by $B$. cepacia $H 111$ requires a functional cep quorum-sensing system. A detailed quantitative analysis of the biofilm structures formed by wild-type and mutant strains suggested that the quorum-sensing system is not involved in the regulation of initial cell attachment, but rather controls the maturation of the biofilm. Furthermore, it is shown that $B$. cepacia is capable of swarming motility, a form of surface translocation utilized by various bacteria to rapidly colonize appropriate substrata. Evidence is provided that swarming motility of $B$. cepacia is quorum-sensing-regulated, possibly through the control of biosurfactant production. Complementation of the cepR mutant H111-R with different biosurfactants restored swarming motility while biofilm formation was not significantly increased. This result suggests that swarming motility per se is not essential for biofilm formation on abiotic surfaces.

Keywords: cystic fibrosis, $\mathrm{N}$-acylhomoserine lactone, biosurfactant

\section{INTRODUCTION}

To date, most research in molecular microbiology has focused on the analysis of planktonic cells in pure cultures and it was not until recently that it was fully appreciated that in nature bacteria predominantly exist as sessile, surface-associated communities, referred to as biofilms (for recent reviews see O'Toole et al., 2000; Tolker-Nielsen \& Molin, 2000; Watnick \& Kolter, 2000). Many persistent and chronic bacterial infections, including periodontitis, otitis media, biliary tract infection and endocarditis, are intrinsically linked to the

Abbreviations: $\mathrm{AHL}, \mathrm{N}$-acylhomoserine lactone; $\mathrm{CF}$, cystic fibrosis; CLSM, confocal laser scanning microscopy; GFP, green fluorescent protein; C8$\mathrm{HSL}, \mathrm{N}$-octanoylhomoserine lactone; C6-HSL, $N$-hexanoylhomoserine lactone; 3-oxo-C6-HSL, N-(3-oxohexanoyl)homoserine lactone. formation of biofilms. Moreover, various medical implants are prone to colonization by pathogenic bacteria (Costerton et al., 1999). Bacterial biofilm infections are particularly problematic as sessile bacteria can withstand host immune responses and are markedly more resistant to antibiotics (up to 1000-fold) and biocides than cells grown in suspension (Schierholz et al., 1999; Xu et al., 2000).

The opportunistic pathogenic bacterium Pseudomonas aeruginosa is capable of chronically colonizing the lungs of patients suffering from cystic fibrosis (CF), the most common lethal inherited disease among the Caucasian population (Koch \& Høiby, 1993; Govan \& Deretic, 1996; Tümmler \& Kiewitz, 1999). During chronic infection, $P$. aeruginosa produces copious amounts of alginate, which forms a matrix completely embedding 
the cells, and becomes highly resistant to antibiotic treatment. These observations led to the suggestion that $P$. aeruginosa may exist as a biofilm in the CF lung (Lam et al., 1980; Costerton et al., 1999). This hypothesis was recently corroborated through profiling of $\mathrm{N}$-acylhomoserine lactone (AHL) signal molecules (Singh et al., 2000). Burkholderia cepacia has been recognized as another important pathogen in patients with CF. Infection with B. cepacia often occurs in patients who are already colonized with $P$. aeruginosa. In fact, it has been suggested that $P$. aeruginosa exoproducts may modify the epithelial cell surface of the lung such that attachment of B. cepacia is facilitated (Saiman et al., 1990). Co-colonization can result in three clinical outcomes: asymptomatic carriage, slow and continuous decline in lung function, or, for approximately $20 \%$ of the patients, fulminant and fatal pneumonia, the socalled 'cepacia syndrome' (Isles et al., 1984).

In both $P$. aeruginosa (for reviews see de Kievit \& Iglewski, 2000; Parsek \& Greenberg, 2000; Williams et al., 2000) and B. cepacia (Lewenza et al., 1999), expression of various virulence factors is controlled by AHL-dependent quorum-sensing systems. These regulatory systems ensure that pathogenic traits are only expressed when the bacterial population density is high enough to overwhelm the host before it is able to mount an efficient response. Interestingly, for $P$. aeruginosa it has been demonstrated that the architecture of biofilms formed on an abiotic surface is also quorum-sensingcontrolled (Davies et al., 1998). These results argue in favour of functional overlaps between factors necessary for biofilm formation and pathogenicity. The quorumsensing system of B. cepacia K56-2 (genomovar III) has been recently identified (Lewenza et al., 1999). This density-dependent regulatory system relies on two proteins: the AHL synthase CepI, which directs the synthesis of $\mathrm{N}$-octanoylhomoserine lactone (C8-HSL) and, as a minor product, $\mathrm{N}$-hexanoylhomoserine lactone (C6-HSL) (Gotschlich et al., 2001), and CepR, which after binding of C8-HSL is thought to activate or repress transcription of target genes. The cep system was demonstrated to positively regulate protease production and to repress synthesis of the siderophore ornibactin (Lewenza et al., 1999). Since the two bacteria not only form mixed biofilms in CF lungs but also utilize the same chemical language, it appears likely that the two species synergistically enhance the others' virulence (McKenney et al., 1995).

The recent development of a simple biofilm assay has greatly facilitated the analysis of the genetic mechanisms underlying biofilm formation. In this assay, bacteria are grown in the wells of microtitre dishes in which the cells attach to the abiotic surface. Following removal of planktonic cells, the established biofilm is quantified after staining with crystal violet. Over the past few years, this assay has been extensively used to identify genes involved in biofilm formation in a number of bacteria, including Escherichia coli (Pratt \& Kolter, 1998), Pseudomonas fluorescens (O’Toole \& Kolter, 1998a), P. aeruginosa (O’Toole \& Kolter, 1998b), Vibrio cholerae (Watnick \& Kolter, 1999) and Streptococcus gordonii (Loo et al., 2000). In the present study we have employed this assay to isolate random transposon insertion mutants in B. cepacia $\mathrm{H} 111$ that are defective in biofilm formation on a polystyrene surface. One of these mutants is demonstrated to bear the transposon within the $c e p R$ gene. This finding prompted us to investigate the role of the cep quorum-sensing system in the strain's ability to form biofilms. It is shown that both biofilm formation and swarming motility are cepregulated phenotypes.

\section{METHODS}

Strains, plasmids and growth conditions. Strains and plasmids used in this study are listed in Table 1. Unless otherwise stated, the strains were grown at $37^{\circ} \mathrm{C}$ in modified LuriaBertani (LB) broth (Andersen et al., 1998) or AB minimal medium (Clark \& Maaløe, 1967) supplemented with $10 \mathrm{mM}$ citrate. Solid media were routinely solidified with $1.5 \%(\mathrm{w} / \mathrm{v})$ agar. Growth media for examination of swimming and swarming motility contained $0.3 \%$ and $0.4 \%$ agar, respectively (Eberl et al., 1996). Antibiotics were added as required at final concentrations of $100 \mu \mathrm{g}$ ampicillin $\mathrm{ml}^{-1}, 10 \mu \mathrm{g}$ tetracycline $\mathrm{ml}^{-1}, 20 \mu \mathrm{g}$ gentamicin $\mathrm{ml}^{-1}$ and $10 \mu \mathrm{g}$ chloramphenicol ml $\mathrm{m}^{-1}$. Kanamycin was used at $50 \mu \mathrm{g} \mathrm{ml}^{-1}$ for E. coli and $100 \mu \mathrm{g} \mathrm{ml}^{-1}$ for $B$. cepacia. Tellurite concentration was $100 \mu \mathrm{g} \mathrm{ml}^{-1}$. For complementation of H111-I, $200 \mathrm{nM} \mathrm{C8-HSL}$ and/or $200 \mathrm{nM}$ C6-HSL were added. Growth of liquid cultures was monitored spectrophotometrically by an Ultrospec Plus spectrophotometer (Pharmacia) by measurement of optical density at $600 \mathrm{~nm}$.

Conjugative plasmid transfer. Plasmids were delivered to $B$. cepacia by triparental mating as described by de Lorenzo \& Timmis (1994). Briefly, donor and recipient strains and also the helper strain E. coli HB101(pRK600) were grown at $37^{\circ} \mathrm{C}$ overnight in $5 \mathrm{ml} \mathrm{LB}$ supplied with the appropriate antibiotics. Following subculturing to an $\mathrm{OD}_{600}$ of $0 \cdot 9$, the cells from $2 \mathrm{ml}$ of culture were harvested, washed and resuspended in $200 \mu \mathrm{l}$ LB. Donor and helper cells $(100 \mu \mathrm{l}$ each) were mixed and incubated for $30 \mathrm{~min}$ at room temperature. Recipient cells $(200 \mu \mathrm{l})$ were added and the mixture was spot-inoculated onto the surface of prewarmed LB agar plates. After overnight incubation at $37^{\circ} \mathrm{C}$, the cells were scraped off and were resuspended in $1 \mathrm{ml} 0.9 \% \mathrm{NaCl}$. Serial dilutions were plated on LB medium containing antibiotics for counter-selection of donor, helper and untransformed recipient cells.

DNA manipulations and nucleotide sequencing. Cloning, restriction enzyme analysis and transformation of E. coli were performed essentially as described by Sambrook et al. (1989). PCR was performed using the TaKaRa rTaq DNA polymerase (TaKaRa Shuzo). Plasmid DNA was isolated with the QIAprep Spin Miniprep kit and chromosomal DNA from $B$. cepacia was purified with the DNeasy Tissue kit. DNA fragments were purified from agarose gels using the QIAquick Gel Extraction kit (all kits were from Qiagen).

For complementation of B. cepacia H111-R, we constructed plasmid pBAH27 $\left(c e p R^{+}\right)$as follows. The cepR gene was PCRamplified using primers cepR-R (5'-GGGGTACCAACCTGACAAGTATGACAGCG-3') and cepR-OV (5'-GGGGTACC GGATGAGCATGGAGAAAAGC-3') (KpnI restriction sites are underlined). Following digestion with $K p n$ I, the PCR fragments were inserted into the broad-host-range vector pBBR1MCS-5 cut with the same enzyme. The plasmid 
Table 1. Bacterial strains and plasmids used in this study

\begin{tabular}{|c|c|c|}
\hline Strain or plasmid & Description & Source or reference \\
\hline \multicolumn{3}{|l|}{ Strains } \\
\hline \multicolumn{3}{|l|}{ E. coli } \\
\hline XL1-Blue & 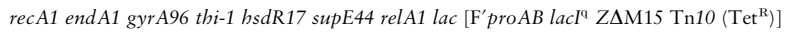 & Stratagene \\
\hline MT102 & $\operatorname{araD139}($ ara-leu $) 7697 \Delta$ lac thi hsdR & Laboratory collection \\
\hline CC118 ( $\lambda$ pir) & $\Delta($ ara-leu $)$ araD $\Delta$ lacX74 galE galK phoA thi-1 rpsE rpoB argE(Am) recA, $\lambda$ pir lysogen & Herrero et al. (1990) \\
\hline \multicolumn{3}{|l|}{ B. cepacia } \\
\hline H111 & CF isolate from a patient at the Medizinische Hochschule Hannover (1993), genomovar III & Römling et al. (1994); Gotschlich et al. (2001) \\
\hline $\mathrm{m} 64$ & CepR :: Tn5 Km2-luxCDABE derivative of H111 & This study \\
\hline H111-I & $\mathrm{Km}^{\mathrm{r}}$, cepI: : Km mutant of $\mathrm{H} 111$ & This study \\
\hline H111-R & $\mathrm{Km}^{\mathrm{r}}$, cepR : : Km mutant of $\mathrm{H} 111$ & This study \\
\hline \multicolumn{3}{|l|}{ Plasmids } \\
\hline pAG & $\mathrm{Gm}^{\mathrm{r}} \mathrm{Km}^{\mathrm{r}}$, pEX18Gm derivative for inactivation of cepI & This study \\
\hline pBAH27 & pBBR1MCS-5 containing the $c e p R$ gene of B. cepacia H111 & This study \\
\hline pBAH33 & $\mathrm{Gm}^{\mathrm{r}} \mathrm{Km}^{\mathrm{r}}$, pEX19Gm derivative for inactivation of $c e p R$ & This study \\
\hline pBBR1MCS-5 & $\mathrm{Gm}^{\mathrm{r}}$, broad-host-range cloning vector & Kovach et al. (1995) \\
\hline pEX18Gm & $\mathrm{Gm}^{\mathrm{r}}$, ori $T^{+} \mathrm{sacB}^{+}$, gene replacement vector with multiple cloning site from pUC18 & Hoang et al. (1998) \\
\hline pEX19Gm & $\mathrm{Gm}^{\mathrm{r}}$, ori $T^{+} s a c B^{+}$, gene replacement vector with multiple cloning site from pUC19 & Hoang et al. (1998) \\
\hline pGEM-3Zf $(+)$ & $\mathrm{Ap}^{r}$, multicopy cloning vector & Promega \\
\hline pMH94 & $A p^{r} \mathrm{Km}^{\mathrm{r}}$, delivery vector for mini-Tn5 Tel $\mathrm{r}^{\mathrm{r}}$ kilAtelAB- $\mathrm{P}_{\mathrm{A} 1 / 04 / 03}-g f p$ mut $3-\mathrm{T}_{\mathrm{o}}-\mathrm{T}_{1}$ & M. Hentzer \& M. R. Parsek (unpublished) \\
\hline pRK600 & $\mathrm{Cm}^{\mathrm{r}}$, ColE1oriV, RK-2Mob ${ }^{+} \mathrm{RK} 2-\mathrm{Tra}^{+}$, helper plasmid in triparental matings & de Lorenzo \& Timmis (1994) \\
\hline pSB403 & 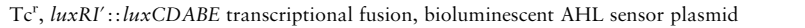 & Winson et al. (1998b) \\
\hline pUTmini-Tn5 Km2-luxCDABE & $\mathrm{Km}^{\mathrm{r}}$, delivery vector for mini $\mathrm{Tn} 5 \mathrm{Km} 2-\operatorname{lux} C D A B E$ & Winson et al. (1998a) \\
\hline
\end{tabular}

containing the insert in the orientation placing the $c e p R$ gene downstream of the $\mathrm{P}_{\text {lac }}$ promoter of the cloning vector was chosen and this construct was designated pBAH27. For flowchamber experiments, the strains were tagged with green fluorescent protein (GFP). This was accomplished by the insertion of a $\mathrm{P}_{\mathrm{A} 1 / 04 / 03^{-}}-\mathrm{ffp}$-T0-T1 transposon cassette (Andersen et al., 1998) into the chromosomes of target strains using the suicide construct pMH94 (M. Hentzer \& M. R. Parsek, unpublished results). Plasmid pMH94 was delivered to target strains by conjugative transfer and integrants were selected on PIA medium (Becton Dickinson Biosciences) containing tellurite.

Sequencing was performed by the dideoxynucleotide chaintermination method (Sanger et al., 1977) in a LI-COR 4200 DNA sequencer. The primer 5'-CAGATCTGATCAAGAGACAG-3', which binds to the I-end of the Tn5 transposon, was used for determination of the transposon insertion point in B. cepacia m64. DNA sequences were compared to other sequences in GenBank using the on-line BLAST search engine at the National Center for Biotechnology Information (http:// www.ncbi.nlm.nih.gov/).

Construction of a $\boldsymbol{B}$. cepacia $\mathbf{H 1 1 1}$ mutant bank. The hybrid transposon mini-Tn $5 \mathrm{Km} 2-l u x C D A B E$ was randomly inserted into the chromosome of $B$. cepacia $\mathrm{H} 111$ by the triparental mating procedure described above. Transconjugants were selected on LB medium containing kanamycin and tetracycline. These random insertion mutants were picked and grown in $150 \mu \mathrm{l} \mathrm{LB}$ medium in the wells of polypropylene MicroWell dishes (Nunc). For storage, $75 \mu \mathrm{l} 50 \%(\mathrm{v} / \mathrm{v})$ glycerol was added and the dishes were frozen at $-80^{\circ} \mathrm{C}$.

Screen for mutants defective in biofilm formation. Biofilm formation in polystyrene microtitre dishes was assayed essentially as described by O'Toole \& Kolter (1998a) and Pratt \& Kolter (1998) with a few modifications. Cells were grown in the wells of the microtitre dishes in $100 \mu \mathrm{l} \mathrm{AB}$ medium supplemented with $10 \mathrm{mM}$ citrate for $48 \mathrm{~h}$ at $30^{\circ} \mathrm{C}$. The medium was then removed and $100 \mu \mathrm{l}$ of a $1 \%(\mathrm{w} / \mathrm{v})$ aqueous solution of crystal violet was added. Following staining at room temperature for $20 \mathrm{~min}$, the dye was removed and the wells were washed thoroughly. For quantification of attached cells, the crystal violet was solubilized in a 80:20 $(\mathrm{v} / \mathrm{v})$ mixture of ethanol and acetone and the absorbance was determined at $570 \mathrm{~nm}$.

Detection and characterization of AHLs. Production of AHLs was investigated with the aid of the bioluminescent plasmid sensor pSB403 (Winson et al., 1998b). This sensor plasmid contains the Photobacterium fischeri luxR gene together with the luxI promoter region as a transcriptional fusion to the bioluminescence genes luxCDABE of Photorhabdus luminescens. The quorum-sensing system of Photobacterium fischeri relies on $\mathrm{N}$-(3-oxohexanoyl)homoserine lactone (3-oxo-C6HSL) and the sensor plasmid consequently exhibits the highest sensitivity for this AHL molecule. However, several other AHL molecules are detected by the sensor, albeit with somewhat reduced sensitivity (Winson et al., 1998b; Geisenberger et al., 2000). Bioluminescence was detected either with the highly sensitive photon-counting camera C2400-40 (Hamamatsu Photonics) or by exposure to an X-ray film. For more detailed analysis, the AHL molecules were extracted from spent culture supernatants of the strains, separated by TLC and AHL spots were visualized by overlaying the TLC plates with soft agar seeded with the sensor strain E. coli MT102(pSB403) as described previously (Shaw et al., 1997; Geisenberger et al., 2000). Routinely, AHLs were extracted twice with dichloromethane (250:100 supernatant/dichloromethane) from $250 \mathrm{ml}$ sterile-filtered supernatants of $B$. cepacia cultures grown in $\mathrm{AB}$ minimal medium containing $10 \mathrm{mM}$ citrate at $30^{\circ} \mathrm{C}$ to an $\mathrm{OD}_{600}$ of $1 \cdot 0$. The combined extracts were dried over anhydrous magnesium sulfate, filtered and evaporated to dryness. Residues were dissolved in $250 \mu \mathrm{l}$ ethyl acetate. Samples $(10 \mu \mathrm{l})$ were then applied to $\mathrm{C}_{18}$ reversed-phase TLC plates (Merck no. 1.15389) and dried with a stream of cold air. Samples were separated by using methanol in water $(60 \%, v / v)$ as the mobile phase. For detection of AHLs, the TLC plate was overlaid with a thin film of LB agar $(143 \mathrm{ml})$ seeded with $7 \mathrm{ml}$ of an exponentially grown AHL biosensor and was then incubated at $30^{\circ} \mathrm{C}$ for $24 \mathrm{~h}$. The tentative identification of AHLs present in spent culture supernatant extracts was achieved by comparison of mobilities $\left(R_{\mathrm{F}}\right.$ values $)$ relative to those for the synthetic AHL standards.

For quantification of AHL signal molecules, $100 \mu \mathrm{l}$ of filter- 


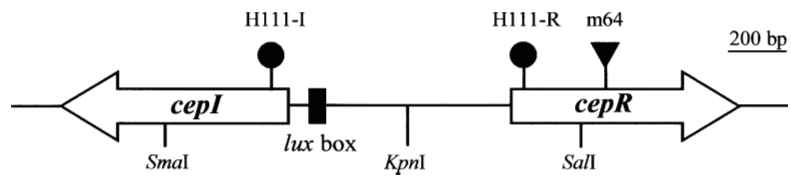

Fig. 1. Physical and genetic map of the cep quorum-sensing region of $B$. cepacia $\mathrm{H} 111$. Genes are marked by open arrows, with the direction of the arrowheads indicating the direction of transcription. A palindrome sequence in the cepl promoter region that is highly homologous to the lux box consensus sequence is indicated. Relevant restriction sites are shown. The location of the mini-Tn5 Km2-luxCDABE element in B. cepacia $\mathrm{m} 64$ is indicated by a triangle. The positions of the npt cassettes in the cep/ mutant $\mathrm{H} 111-\mathrm{I}$ and the cepR mutant H111-R are indicated by circles.

sterilized supernatants of cultures grown in LB medium to an $\mathrm{OD}_{600}$ of 3.0 was added to $100 \mu \mathrm{l}$ of an exponential culture of E. coli MT102(pSB403) in the wells of a FluoroNunc Polysorp microtitre dish. Following incubation at $30^{\circ} \mathrm{C}$ for $6 \mathrm{~h}$, bioluminescence was measured with a Lamda Fluoro 320 Plus reader (Bio-Tek Instruments).

Exoenzyme and siderophore production. Exoenzyme and siderophore production was tested by streaking strains on appropriate indicator plates. Proteolytic activity was determined on LB medium supplemented with $2 \%$ skim milk, chitinolytic activity on ethylene glycol chitin agar (Connell et al., 1998) and lipolytic activity on tributyrin agar base containing $1 \%$ glycerol tributyrate (both Merck). Clear haloes around the colonies after incubation at $37^{\circ} \mathrm{C}$ overnight indicated exoenzyme activity. Siderophore production was tested by growing strains on CAS agar (Schwyn \& Neilands, 1987) for $24 \mathrm{~h}$. Siderophores remove the iron from the CAS dye complex, resulting in a colour change around the colonies from blue to orange.

Construction of $B$. cepacia $\mathrm{H} 111$ cepl and cepR mutant strains. Defined cep mutants (Fig. 1) were constructed by the gene replacement method described by Hoang et al. (1998). For the construction of a cepR mutant, two DNA fragments were PCR-amplified: an $850 \mathrm{bp}$ EcoRI-SacI fragment spanning the intergeneric region plus the first $75 \mathrm{bp}$ of $c e p R$ using the primer pair intercep-f-Eco (5'-GGAATTCGAGATCCGCCGCGAGTTCG-3') and intercep-r-Sac (5'-GATCCGCTGGAAGAGCTCC-3'), and a 760 bp SphI-HindIII fragment containing the $3^{\prime}$ region of the cepR gene using the primer pair cepR-f-Sph (5'-ACATGCATGCGCTCGGATTCGAATACTGC-3') and cepR-r-Hind (5'-CCCAAGCTTAGAAGCTCGAGCAGATCGC-3'). Using the restriction sites introduced by the PCR primers (respective sites are underlined), these two DNA fragments were successively inserted into the compatible sites of the gene replacement vector pEX19Gm (Hoang et al., 1998). Next, the $n p t$ gene from transposon Tn903 (Oka et al., 1981), which confers resistance to kanamycin, was cloned as a $1.7 \mathrm{~kb}$ Bam HI fragment into the vector cut with the same enzyme. The final construct, which was designated pBAH33, was transferred to B. cepacia $\mathrm{H} 111$ and integrants were selected on LB medium containing kanamycin and tetracycline. To screen for gene replacement mutants, $\mathrm{Kan}^{\mathrm{r}}$ clones were tested for gentamicin sensitivity as the gentamicin resistance gene is lost in the case of a double crossover event. One mutant, which was designated B. cepacia H111-R, was chosen and the correct genetic structure of the strain was confirmed by Southern Blot analysis. Construction of a defined cepI mutant was performed as described for the cepR mutant, except that the gene replacement vector $\mathrm{pEX} 18 \mathrm{Gm}$ and two different PCR fragments were used. An 800 bp EcoRI-SacI fragment spanning the intergeneric region plus $100 \mathrm{bp}$ of $c e p R$ was amplified with the primer pair igR-f-Eco (5'-GGAATTC CCAGTATTCGAATCCGAGCCGC-3') and igR-r-Sac (5'CGAGCTCGGGATGTCCTCGGATCTGTGC3'), and a $650 \mathrm{bp} \mathrm{Bam} \mathrm{HI}-H$ indIII fragment containing the $5^{\prime}$ region of cepI was amplified using the primer pair cepI-f-Bam $\left(5^{\prime}-\right.$ CGGGATCCCGCCTTCGTTCACGAGGAAGGG-3') and cepI-r-Hind (5'-CCCAAGCTTGGGCGCGCGTTCCGGCTCAGG-3'). The final gene replacement construct was designated pAG and the respective B. cepacia H111 cepI mutant was named H111-I.

Monitoring of biofilm formation by confocal laser scanning microscopy (CLSM) and image analysis. Biofilms were grown in artificial flow cells supplied with $\mathrm{AB}$ medium and containing $1 \mathrm{mM}$ glucose (ABG). The flow system was assembled and prepared as described previously (Christensen et al., 1999). The substratum consisted of a microscope glass coverslip (Knittel Gläser). Overnight cultures in ABG medium were subcultured to an $\mathrm{OD}_{600}$ of 0.7 before dilution in $0.9 \% \mathrm{NaCl}$ to an $\mathrm{OD}_{600}$ of $0 \cdot 1$. Aliquots $(300 \mu \mathrm{l})$ of these dilutions were used to inoculate the flow channels. Medium flow was kept at a constant rate of $0.7 \mathrm{~mm} \mathrm{~s}^{-1}$ by a Watson-Marlow $205 \mathrm{~S}$ peristaltic pump. Incubation temperature was $30^{\circ} \mathrm{C}$.

Microscopic inspection and image acquisition were performed on a confocal laser scanning microscope (TCS4D; Leica Lasertechnik) equipped with a $63 \times / 1.32-0.6$ oil objective. For statistical evaluation of biofilm structures, a $40 \times / 0.75$ air objective was used. Image scanning was carried out with the $488 \mathrm{~nm}$ laser line of an $\mathrm{Ar} / \mathrm{Kr}$ laser. Captured images were visualized using the IMARIS software package (Bitplane) running on a Silicon Graphics Indigo 2 workstation.

For statistical evaluation of biofilm structures, three independent rounds of biofilm experiments were performed, and in each round, each strain was grown in two separate channels. Seven image stacks were taken of each channel every $24 \mathrm{~h}$ for $7 \mathrm{~d}$ after inoculation. These images were analysed by the computer program COMSTAT, which comprises various features for quantifying three-dimensional biofilm image stacks (Heydorn et al., 2000). The parameters used for characterization of biofilm architecture included biomass, substratum coverage, mean thickness and roughness coefficient.

\section{RESULTS}

\section{Screening for biofilm-defective mutants}

To determine the optimum experimental conditions for attachment of $B$. cepacia H111 to abiotic surfaces, biofilm assays were carried out under various conditions. While the strain formed thick biofilms in polystyrene microtitre dishes, no attachment was observed in dishes made of polypropylene. In contrast to other bacteria (O'Toole \& Kolter, 1998a; Danese et al., 2000; Loo et al., 2000), biofilm formation by B. cepacia H111 is virtually independent of medium composition. AB minimal medium supplemented with glucose or citrate supported surface colonization as well as rich media such as LB. Furthermore, incubation temperatures ranging from 30 to $37^{\circ} \mathrm{C}$ did not significantly affect biofilm yields (data not shown). For routine biofilm assays we used $\mathrm{AB}$ minimal medium containing $10 \mathrm{mM}$ citrate and incubated the microtitre dishes for $48 \mathrm{~h}$ at $30^{\circ} \mathrm{C}$. 


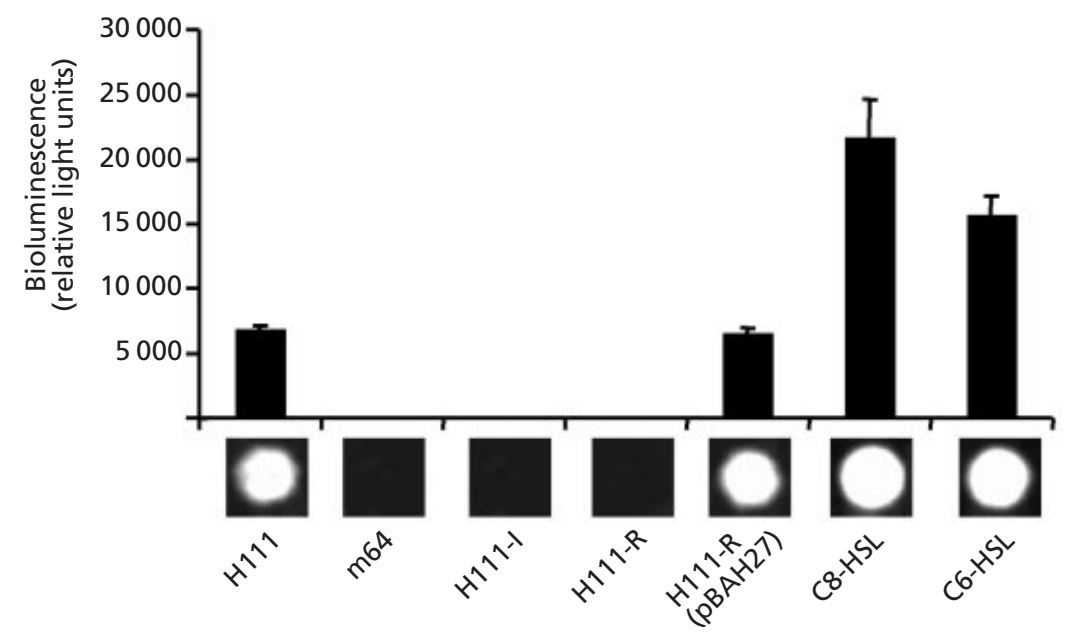

Fig. 2. AHL production by $B$. cepacia $\mathrm{H} 111$ is subject to positive feedback regulation. Aliquots $(100 \mu \mathrm{l})$ of filter-sterilized culture supernatants of wild-type H111, the cepl mutant H111-I, the cepR mutants m64 and $\mathrm{H} 111-\mathrm{R}$ and the complemented $\operatorname{cep} R$ mutant H111-R(pBAH27) were mixed with an exponential culture of the AHL sensor MT102(pSB403) in a microtitre dish. Following incubation for $6 \mathrm{~h}$, bioluminescence was quantified with a luminometer. Error bars represent the standard deviation of the mean for four independent wells. Bioluminescence was also visualized with the aid of a photon camera (pictures shown below the $x$ axis). C8-HSL $(10 \mu \mathrm{M})$ and C6-HSL $(1 \mu \mathrm{M})$ standards were included as controls.

Table 2. Phenotypic characterization of $B$. cepacia $\mathrm{H} 111$ and cep-defective mutants

Production of AHLs was determined by testing culture supernatants for stimulation of the bioluminescent sensor plasmid pSB403. Synthesis of extracellular hydrolytic enzymes and siderophore activities were assessed by streaking strains on appropriate indicator plates as described in Methods. Swimming and swarming motility was tested on media solidified with $0 \cdot 3 \%$ and $0 \cdot 4 \%$ agar, respectively. Wild-type $\mathrm{H} 111$, the cepR mutant H111-R, the complemented cepR mutant H111-R(pBAH27) and the cepI mutant H111-I in the presence or absence of C8-HSL $(200 \mathrm{nM})$ were investigated. +, Activity exhibited by the wild-type; $(+)$ significantly reduced activity; - , no detectable activity; NA, not applicable.

\begin{tabular}{|lccccc|}
\hline Phenotype & H111 & H111-R & $\begin{array}{c}\text { H111-R } \\
\text { (pBAH27) }\end{array}$ & H111-I & $\begin{array}{c}\text { H111-I } \\
+ \text { AHL }\end{array}$ \\
\hline AHL & + & - & + & - & NA \\
Protease & + & - & + & - & + \\
Chitinase & + & $(+)$ & + & $(+)$ & + \\
Lipase & + & + & + & + & + \\
Siderophore & + & $(+)$ & + & $(+)$ & + \\
Swarming & + & - & + & - & + \\
Swimming & + & + & + & + & + \\
\hline
\end{tabular}

The suicide vector pUT (de Lorenzo \& Timmis, 1994) was used to deliver the hybrid transposon miniTn5 Km2-luxCDABE (Winson et al., 1998a) into the $B$. cepacia $\mathrm{H} 111$ chromosome. A collection of 5000 random insertion mutants was screened for ability to form biofilms. A total of eighteen mutants which were to different degrees defective in biofilm formation was obtained (data not shown). During the course of a detailed phenotypical characterization of these mutants we noticed that one, $\mathrm{m} 64$, was deficient in the production of AHL signal molecules. As shown in Fig. 2, the wildtype strain H111 strongly activated the bioluminescent AHL sensor plasmid pSB403 while no activation was observed with m64. This result is reminiscent of the situation found with $P$. aeruginosa, for which it has been shown that development of a mature biofilm is quorumsensing-regulated (Davies et al., 1998). We therefore focused our further investigations on the analysis of this mutant.

Since B. cepacia H111 produces two AHL molecules, C8-HSL and C6-HSL, in a ratio of 10:1 (Gotschlich et al., 2001), we next extracted the signal molecules from spent culture supernatants of the wild-type and the mutant and analysed them by TLC. This analysis showed that the mutant is impaired in the production of both molecules (data not shown). Addition of $200 \mathrm{nM}$ C8-HSL to the growth medium did not restore biofilm formation (data not shown). To further characterize the nature of the transposon insertion in strain $\mathrm{m} 64$, the mutated locus was cloned into the cloning vector pGEM$3 Z \mathrm{Zf}(+)$ as a $S p h \mathrm{I}$ fragment, selecting for transposonencoded kanamycin resistance. The resulting plasmid contains part of the transposon and approximately $3 \mathrm{~kb}$ of chromosomal DNA upstream of the transposon insertion point. Nucleotide sequence analysis of the flanking DNA revealed that the transposon had inserted into the cepR gene of $B$. cepacia $\mathrm{H} 111$ at position 356 (Fig. 1; GenBank accession no. AF330020). These data are fully consistent with previous results that showed that expression of cepI and thus AHL signal production is regulated by CepR (Lewenza et al., 1999). More importantly, these results suggest that the cep quorumsensing system is involved in the regulation of biofilm formation in B. cepacia H111.

\section{Construction and characterization of defined B. cepacia H111 cep mutants}

To investigate the role of the cepIR genes in biofilm formation in greater detail, we constructed site-directed insertion mutations in the two genes by using a gene replacement method (see Methods for details and Fig. 1). The genetic structure of the two mutants, which were designated H111-I and H111-R, respectively, was confirmed by Southern blot analysis (data not shown).

As expected, neither the cepI mutant H111-I nor the $c e p R$ mutant H111-R produced detectable amounts of 
(a)
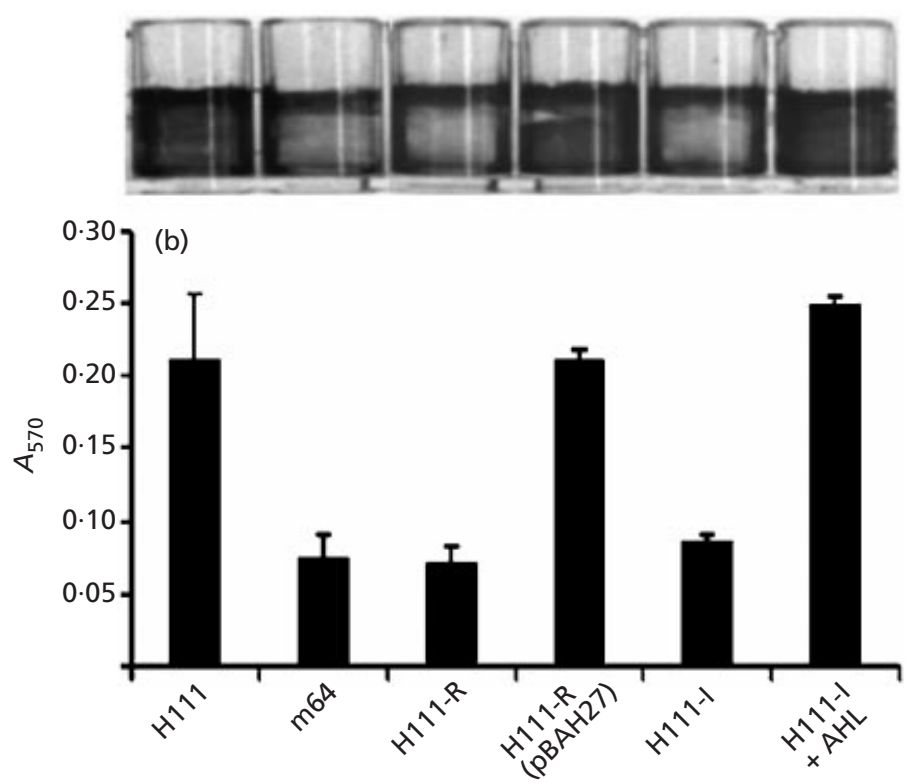

Fig. 3. The cep quorum-sensing system controls biofilm formation by $B$. cepacia $H 111$. Strains were grown in $A B$ minimal medium supplemented with $10 \mathrm{mM}$ citrate in the wells of polypropylene microtitre dishes. After incubation for $48 \mathrm{~h}$ at $30^{\circ} \mathrm{C}$, planktonic cells were removed and attached cells were stained with crystal violet. Biofilms formed by wild-type $\mathrm{H} 111$, the cepR mutants m64 and H111-R, the complemented cepR mutant H111-R(pBAH27) and the cepl mutant $\mathrm{H} 111-\mathrm{I}$ in the presence or absence of C8-HSL $(200 \mathrm{nM})$ are shown in (a); quantification of the biofilm-associated dye is shown in (b). Error bars represent the standard deviation of the mean for six independent wells.
AHLs (Fig. 2). However, production of AHLs was restored to wild-type levels when H111-R was complemented with plasmid pBAH27, which contains the cepR gene inserted into the broad-host-range vector pBBR1MCS-5.

B. cepacia produces different siderophores and a number of exoenzymes that are thought to be pathogenesis factors in humans as well as in plants (Lonon et al., 1988; McKevitt et al., 1989; Gessner \& Mortensen, 1990; Yohalem \& Lorbeer, 1994; Darling et al., 1998). In a recent study it was shown that the cep system of $B$. cepacia $\mathrm{K} 56-2$ is involved in the regulation of the synthesis of extracellular enzymes and siderophores (Lewenza et al., 1999). We therefore tested the B. cepacia H111 wild-type and the two mutants H111-I and H111$\mathrm{R}$ for the production of extracellular protease, lipase, chitinase and siderophores on appropriate indicator plates. The results of these investigations are summarized in Table 2. Consistent with the results reported by Lewenza et al. (1999), both mutants showed a clear reduction in protease activity. Furthermore, proteolytic activities of the mutants were completely restored when mutant H111-I was grown in the presence of $200 \mathrm{nM}$ C8-HSL or when plasmid pBAH27 $\left(c e p R^{+}\right)$was transferred to mutant H111-R. Both mutants were found to produce significantly lowered amounts of siderophores as assessed on CAS indicator plates. As for proteolytic activity, these defects were restored to wild-type levels by the external addition of $200 \mathrm{nM} \mathrm{C8-HSL}$ to H111-I or by complementation of H111-R with plasmid pBAH27 $\left(c e p R^{+}\right)$. These data are in contrast to the results of the above-mentioned study, which showed that inactivation of either cepI or cepR results in an up-regulation of siderophore production in B. cepacia K56-2. Most likely, this apparent discrepancy can be attributed to the different strains used in the studies. Likewise, while Lewenza et al. (1999) observed reduced lipase activity with the $c e p R$ but not with the cepI mutant of K56-2, we were unable to detect any difference in the lipase activities of H111, H111-I and H111-R. Chitinase activity was slightly reduced in the two mutants when compared with the wild-type, and since complementation (as described above) restored the defects we suggest that chitinase production in B. cepacia H111 is, at least in part, regulated by quorum sensing.

\section{The cep system of $B$. cepacia H111 controls biofilm maturation}

We next tested the two mutants H111-I and H111-R for their abilities to form biofilms in microtitre dishes as described above. In agreement with our initial observation with strain $\mathrm{m} 64$, we found that both mutants were defective in biofilm formation (Fig. 3). However, addition of $200 \mathrm{nM}$ C8-HSL to the medium completely restored biofilm formation by mutant H111-I. Likewise, H111-R harbouring plasmid pBAH27 formed wild-type biofilms. As one reason for lowered biofilm formation capability is reduced growth, we determined the growth rates of H111, H111-I and H111-R in AB minimal medium supplemented with $10 \mathrm{mM}$ citrate. Wild-type and mutant strains were found to grow equally well in liquid culture (data not shown).

To further analyse the role of the cep system in biofilm formation, we employed artificial flow cells, which allow biofilm development to be followed on a glass surface under highly defined conditions in real time (Christensen et al., 1999). Moreover, by the use of a confocal laser scanning microscope the three-dimensional structure of the biofilm can be reconstructed. For this analysis, it was necessary to tag B. cepacia H111 and the two mutants H111-I and H111-R with GFP. This was accomplished by inserting a $\mathrm{P}_{\mathrm{A} 1 / 04 / 03}-g f p-T 0-\mathrm{T} 1$ transposon cassette (Andersen et al., 1998) randomly 


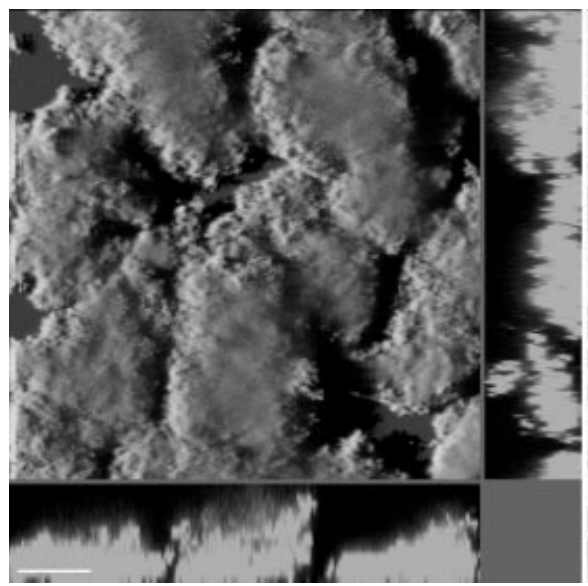

$\mathrm{H} 111$

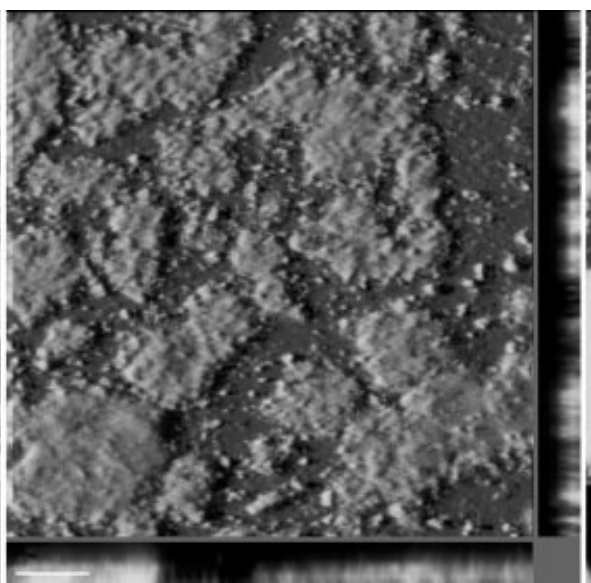

H111-I

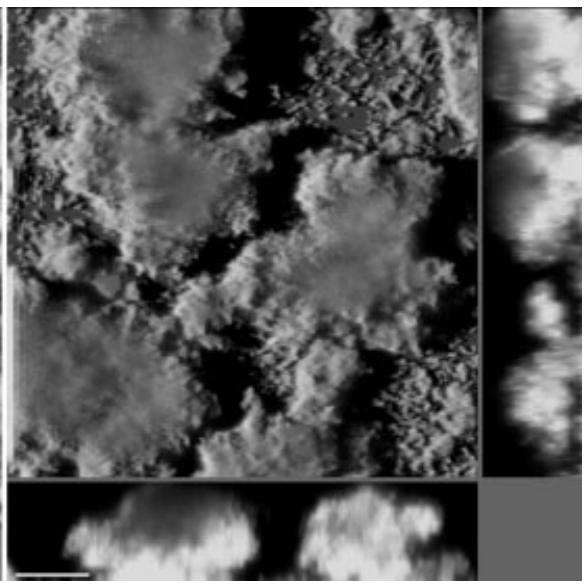

$H 111-I+A H L$

Fig. 4. Biofilm formation by $B$. cepacia $\mathrm{H} 111$ is quorum-sensing-regulated. Flow chambers were inoculated with gfptagged derivatives of wild-type $\mathrm{H} 111$ and of the cep/ mutant H111-I in the presence or absence of $200 \mathrm{nM}$ C8-HSL. The CLSM pictures shown were taken $4 \mathrm{~d}$ after inoculation. The larger central plots are simulated fluorescence projections. Shown in the right and lower frames are vertical sections through the biofilms. Bars, $20 \mu \mathrm{m}$.

into the chromosome of each of the three strains using the suicide plasmid pJMT6 (Sanchez-Romero et al., 1998). The tagged strains used for further investigations were carefully tested with respect to growth rates in liquid medium and biofilm formation in microtitre dishes and were found to be indistinguishable from the parental strains (data not shown).

Parallel flow chambers were inoculated with each of the three GFP-tagged strains and biofilm development was monitored on a daily basis for $7 \mathrm{~d}$. Visual inspection of CLSM images revealed that the biofilms formed by the two mutants not only differed in their substratum coverage and thickness, as had been anticipated from the microtitre plate assays, but also exhibited strikingly different structures (Fig. 4 and data not shown). Both wild-type and mutant strains formed characteristic microcolonies after initial surface attachment. However, while wild-type biofilms rapidly matured and covered most of the available surface space within $24 \mathrm{~h}$, mutant biofilms were arrested in the microcolony stage and never colonized the entire surface during the course of the experiment.

To more accurately describe the differences in the biofilms formed by the wild-type and the cep mutants, we employed the computer program сOMSTAT, which was recently developed for the quantitative characterization of biofilm structures (Heydorn et al., 2000). Out of ten image analysis features which the program provides for quantifying three-dimensional image stacks acquired by CLSM, we chose the following: biomass, mean thickness, substratum coverage and roughness coefficient. To generate data of statistical value, three independent rounds of biofilm experiments were performed. In each round, two flow chamber channels were inoculated with each strain tested. Seven image stacks per channel were taken every $24 \mathrm{~h}$ for 7 days and these were analysed with COMSTAT (Heydorn et al., 2000). In full agreement with the visual impression, the coefficients for mean thickness and for biomass were greatly reduced for the cep mutants when compared with the wild-type (Fig. 5). The two mutants colonize the surface less efficiently than the wild-type, a fact that is reflected by a higher value for substratum coverage for the latter strain. The roughness coefficient is a measure of the variance of biofilm thickness and the higher values of this coefficient for mutant biofilms indicate that they are more heterogeneous than wild-type biofilms. Importantly, in the presence of $200 \mathrm{nM}$ C8-HSL, strain H111I forms a biofilm that is completely indistinguishable from the one of the wild-type strain (Fig. 4 and Fig. 5a). Likewise, strain H111-R harbouring plasmid pBAH27 $\left(c e p R^{+}\right)$forms a biofilm with a typical wild-type structure (Fig. 5b).

In conclusion, these results clearly show that the cep quorum-sensing system of B. cepacia $\mathrm{H} 111$ is involved in the control of biofilm formation. More specifically, our data suggest that the cep system may not be important for the initial attachment of cells to the surface but is essential for the differentiation of microcolonies, a process that is required for the development of a mature biofilm.

\section{Swarming motility of $B$. cepacia $\mathrm{H} 111$ is regulated by the cep system}

Genetic studies have shown that the formation of a mature biofilm proceeds through an ordered series of steps (for recent reviews see Pratt \& Kolter, 1999; Watnick \& Kolter, 2000; O’Toole et al., 2000). In this model, motility plays a major role in biofilm formation. Flagella-mediated motility is believed to be required to overcome repulsive forces at the surface of the substratum. Furthermore, once the initial contact to the surface is established, cells are thought to move on top 

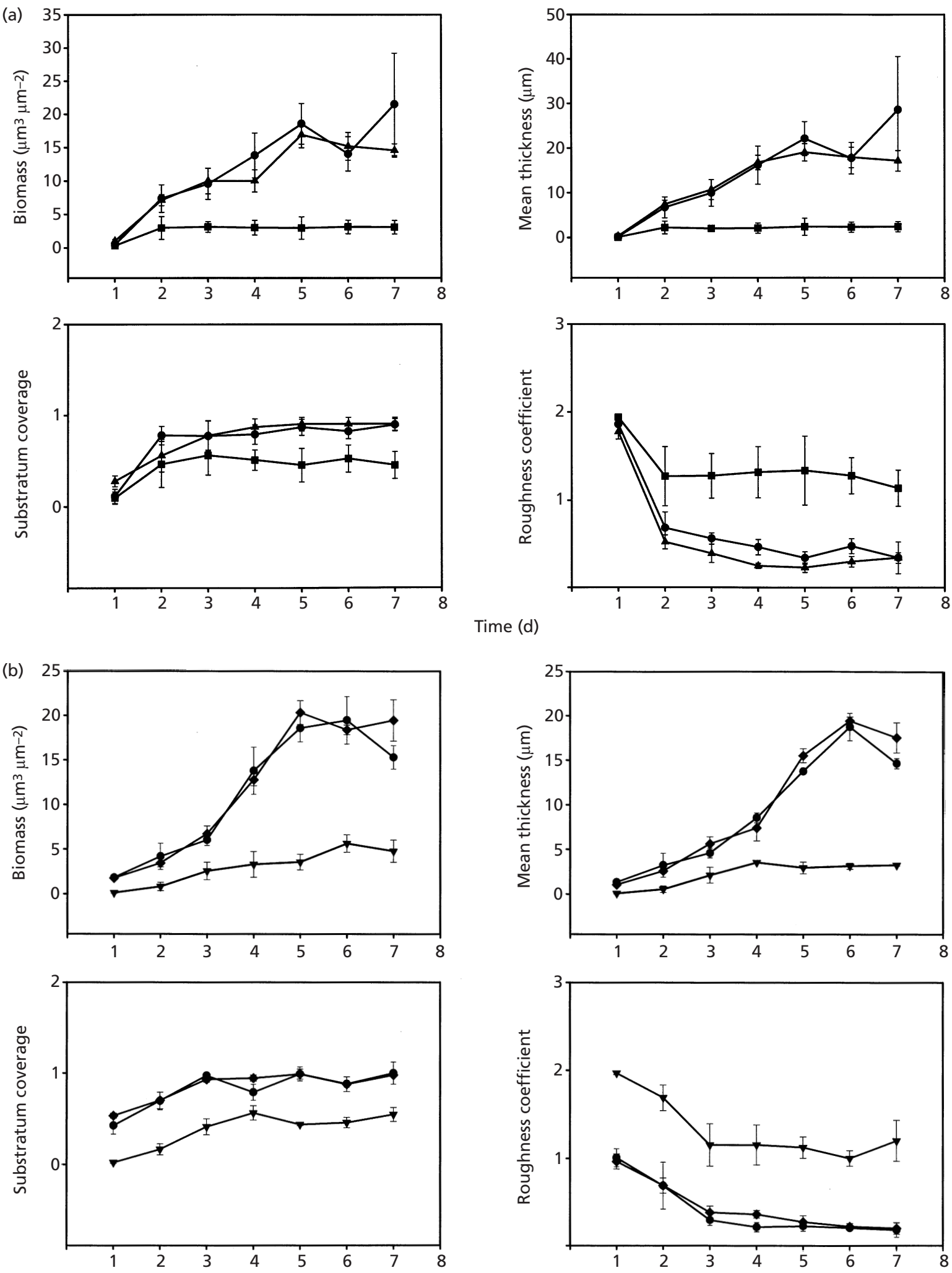

Time (d)

Fig. 5. Quantification of biofilm structures. Biofilms of wild-type $H 111(0)$, and (a) the cepl mutant $H 111-I$ in the presence $(\boldsymbol{\Delta})$ or absence $(\boldsymbol{\square})$ of $200 \mathrm{nM} \mathrm{C8-HSL}$, and (b) the cepR mutant H111-R $(\boldsymbol{\nabla})$ and the complemented cepR mutant H111-R(pBAH27) ( $\diamond)$ were grown in artificial flow cells. CLSM pictures were taken daily for 1 week and these pictures were analysed by the computer program COMSTAT (Heydorn et al., 2000). Parameters calculated were biomass, mean biofilm thickness, substratum coverage and roughness coefficient. Mean values for seven independent CLSM pictures are shown with standard deviations. 


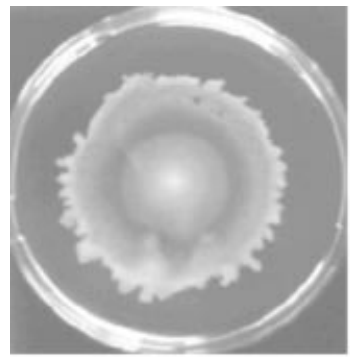

H111

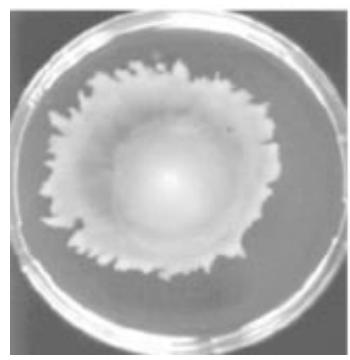

H111-R(pJBA27)

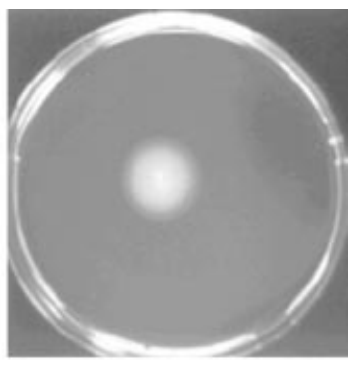

H111-R

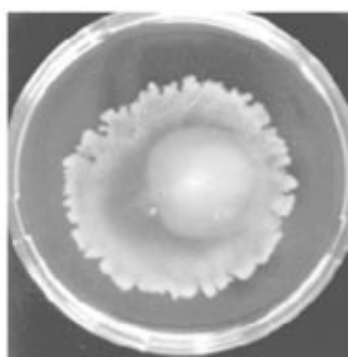

$H 111-R+W 2$
Fig. 6. Swarming motility of $B$. cepacia $\mathrm{H} 111$ is quorum-sensingregulated. Swarming of wild-type $\mathrm{H} 111$, the cepR mutant $\mathrm{H} 111$ $R$ in the presence or absence of the biosurfactant serrawettin W2 $\left(1 \mu \mathrm{g} \mathrm{ml}^{-1}\right)$ and the complemented cepR mutant H111$\mathrm{R}(\mathrm{pBAH} 27)$ was tested on minimal medium solidified with $0.4 \%$ agar and supplemented with $0.1 \%$ Casamino acids. All strains exhibited limited swimming motility at the point of inoculation under the assay conditions used.

of the substratum to form microcolonies. Finally, these microcolonies undergo a differentiation process which leads to the development of a typical three-dimensional biofilm architecture.

For $P$. aeruginosa, it has been shown that aggregation of the cells to microcolonies is dependent on twitching motility, a special form of surface translocation that depends on type IV pili (O'Toole \& Kolter, 1998b). For $V$. cholerae El Tor and E. coli, it has been suggested that flagella-driven motility is not only important for initial attachment of cells to the substratum but also for translocation along the surface in a process that leads to the formation of microcolonies (Pratt \& Kolter, 1998; Watnick \& Kolter, 1999). The importance of motility for biofilm formation, together with the fact that different forms of bacterial motility, including swimming of Yersinia pseudotuberculosis (Atkinson et al., 1999), twitching of $P$. aeruginosa (Glessner et al., 1999) and swarming of Serratia liquefaciens (Eberl et al., 1996) and $P$. aeruginosa (Köhler et al., 2000) are quorumsensing-regulated, prompted us to investigate whether the cep system of B. cepacia H111 is involved in the control of motility.

When cells of B. cepacia $\mathrm{H} 111$ are point-inoculated into $\mathrm{AB}$ minimal medium which is supplemented with $10 \mathrm{mM}$ citrate and solidified with $0.3 \%$ agar, they swim through the water channels in the agar giving rise to typical chemotactic rings. Swimming behaviour of the cep mutants and the wild-type was completely indistinguishable, indicating that swimming motility is not quorum-sensing-regulated (data not shown). We also tested the strain for twitching motility under various conditions, but were unable to demonstrate this form of motility for strain H111. However, during the course of these experiments we observed that, when medium containing $0.4 \%$ agar was supplemented with $0 \cdot 1 \%$ Casamino acids, cells also spread as a thin layer on the top of the agar surface. Microscopic inspection revealed that the cells migrate in a co-ordinated fashion that is characteristic of swarming motility (for reviews see Allison \& Hughes, 1991; Harshey, 1994; Eberl et al., 1999). The migration front of the expanding colony is preceded by a visible layer of slime-like material giving the colony a glistening appearance, a phenomenon that is typical for this form of motility. After incubation for 36 h, B. cepacia H111 colonized the entire surface of the agar plate (Fig. 6). By contrast, the two cep mutants were unable to swarm. Moreover, the mutants were also deficient in the production of extracellular slime. Addition of $200 \mathrm{nM}$ C8-HSL to the medium restored both swarming motility and slime production of mutant H111-I (data not shown). Both phenotypes were also restored when mutant H111-R was complemented with plasmid pBAH27 (cepR $R^{+}$) (Fig. 6). These results show that swarming motility of B. cepacia H111 is under control of the cep quorum-sensing system. Previously, it has been shown that AHL-mediated cell-cell communication is also required for swarming motility of $S$. liquefaciens MG1 (Eberl et al., 1996) and P. aeruginosa (Köhler et al., 2000). In both bacteria the quorumsensing systems control the production of biosurfactants, namely rhamnolipids in the case of $P$. aeruginosa and serrawettin W2 in the case of $S$. liquefaciens, which are essential for swarming motility (Ochsner \& Reiser, 1995; Lindum et al., 1998; Köhler et al., 2000). A S. liquefaciens mutant defective in the synthesis of AHL molecules is unable to swarm unless the medium is supplemented with either AHLs or a compound capable of lowering the surface tension of the medium such as serrawettin W2, surfactin or trace amounts of SDS (Lindum et al., 1998; Eberl et al., 1999). We therefore tested the two cep mutants of B. cepacia for their ability to swarm on low-agar plates supplemented with either surfactin or serrawettin W2. The two cep mutants swarmed on this medium (Fig. 6 and data not shown), suggesting that production of a biosurfactant in $B$. cepacia $\mathrm{H} 111$ is controlled by the cep quorum-sensing system, which in turn is required for swarming motility of this bacterium.

\section{Swarming motility is not required for biofilm formation}

For Vibrio parahaemolyticus, it has been demonstrated that swarming plays an important role in attachment and colonization of chitinaceous shells of crustaceans (Belas \& Colwell, 1982). To investigate whether swarming motility is involved in the process of biofilm formation by B. cepacia, we tested the wild-type and the 
two cep mutants for their abilities to form biofilms in minimal medium supplemented with different amounts of surfactin and serrawettin. The presence of high concentrations of the surfactants in the medium completely prevented the cells from attaching to the surface of the microtitre dishes. Importantly, the surfactant concentrations used did not influence the growth rates of the strains in liquid medium (data not shown). At lower concentrations, which were sufficiently high to completely restore swarming behaviour of the mutants, the two surfactants very weakly (less than $10 \%$ increase in $A_{570}$ ) but reproducibly stimulated biofilm formation by the cep mutants while their presence did not affect biofilm formation by the wild-type. However, these results suggest that swarming motility per se is unlikely to play a major role in biofilm formation.

\section{DISCUSSION}

AHL-dependent communication systems provide bacteria with a regulatory mechanism that enables individual cells to sense their own population density. In response to the size of the population, i.e. when a certain critical mass, the 'quorum' has been attained, cells collectively induce the expression of particular phenotypic traits, which are not observable with individual cells. Hence, quorum-sensing can be viewed as an example of primitive multicellular behaviour. In nature, bacteria are normally associated with surfaces, on which they form highly structured biofilms (Costerton et al., 1995; Davey \& O’Toole, 2000; Tolker-Nielsen \& Molin, 2000; O'Toole et al., 2000). Bacteria living in biofilms are embedded in a matrix of extracellular polymeric substances and thus cell densities are obviously extremely high in these surface-attached communities. By contrast, bacteria growing planktonically, for example in the water column of aquatic systems, only rarely reach high cell densities. It is therefore conceivable that quorum-sensing is a particularly valuable mechanism for gene regulation in biofilms. In support of this view, it has been recently demonstrated that AHL molecules are present both in natural biofilms growing on submerged stones in a river and in biofilms formed on urethral catheters (McLean et al., 1997; Stickler et al., 1998). Moreover, for P. aeruginosa it was shown that the las quorum-sensing system is directly involved in the regulation of biofilm formation (Davies et al., 1998). When compared with the wild-type, a lasI mutant of $P$. aeruginosa only forms flat and undifferentiated biofilms, suggesting that the las system is in some way required for the maturation of biofilms. Importantly, the lasI mutant biofilm exhibited greater sensitivity to the biocide SDS in comparison to the wildtype biofilm. The results presented for biofilm formation by $B$. cepacia $\mathrm{H} 111$ are fully consistent with this hypothesis. Mutants defective in the cep quorum-sensing system form microcolonies on a glass surface that are indistinguishable from those formed by the wild-type, indicating that the early events of biofilm formation are unaffected by the mutations. Similar to the lasI mutant of $P$. aeruginosa, however, these microcolonies are unable to differentiate and thus failed to develop into a mature biofilm.

During an attempt to identify cep-regulated factors required for biofilm formation by $B$. cepacia, we noticed that the strain's ability to swarm on suitable surfaces is quorum-sensing-regulated. Swarming motility is an intrinsically surface-dependent mode of translocation, which, to the best of our knowledge, has not been reported earlier for members of the genus Burkholderia but has been described for many other bacteria (Allison \& Hughes, 1991; Harshey, 1994). Swarming motility of quorum-sensing-defective mutants of $B$. cepacia $\mathrm{H} 111$ could be fully restored by supplementing the media with different surfactants. We therefore propose that the cep system of $B$. cepacia controls the production of a biosurfactant, which is required for swarming motility of the strain. Noteworthy in this context is the recent finding that another genomovar III B. cepacia strain (J2315) produces a lipopeptide of unknown structure which exhibits strong surface-active properties (Hutchison et al., 1998). Our results are reminiscent of the situation found with $S$. liquefaciens and $P$. aeruginosa. These two bacteria have been demonstrated to employ quorum-sensing systems to control the synthesis of the surface-active compounds serrawettin W2 and rhamnolipids, respectively (Ochsner \& Reiser, 1995; Lindum et al., 1998). Since the ability to swarm is strictly dependent on the production of biosurfactants, swarming motility is a quorum-sensing-regulated phenomenon in both bacteria.

The addition of surfactants to the medium, at concentrations sufficiently high to restore swarming motility of the cep mutants to the level of the wild-type, only weakly, if at all, increased biofilm formation by the $B$. cepacia H111 cep mutants. This suggests that locomotion via swarming motility is not required for biofilm formation. On the other hand, the cep-regulated production of the biosurfactant itself may affect biofilm formation as previous results have demonstrated that various surface-active compounds have the capability of regulating the attachment and detachment of bacteria to and from surfaces (Rosenberg \& Ron, 1999; Ahimou et al., 2000). There are several possible explanations as to why our attempts to substitute the missing biosurfactant with surfactin, serrawettin W2 or SDS failed to restore biofilm formation by the cep mutants: (i) the physical properties of the surfactants used and the one produced by the strain are substantially different, (ii) production of the surfactant has to follow a specific temporal and/or spatial expression pattern within the biofilm, or (iii) other cep-regulated, as yet unidentified, factors may be required for biofilm formation. Work currently under way aims to test these possibilities.

\section{ACKNOWLEDGEMENTS}

We thank B. Schumacher for excellent technical assistance, B. Tümmler, H. P. Schweitzer and M. E. Kovach for providing 
bacterial strains and plasmids, and P. Williams for the generous gift of synthetic AHLs.

This work was supported by grants from the BMBF and the DFG (EB 2051/1-2) to L.E. and the Danish Medical Research Council to M.G.

\section{REFERENCES}

Ahimou, F., Jacques, P. \& Deleu, M. (2000). Surfactin and iturin A effects on Bacillus subtilis surface hydrophobicity. Enzyme Microb Technol 27, 749-754.

Allison, C. \& Hughes, C. (1991). Bacterial swarming: an example of prokaryotic differentiation and multicellular behaviour. Sci Prog 75, 403-422.

Andersen, J. B., Sternberg, C., Poulsen, L. K., Petersen Bjørn, S., Givskov, M. \& Molin, S. (1998). New unstable variants of green fluorescent protein for studies of transient gene expression in bacteria. Appl Environ Microbiol 64, 2240-2246.

Atkinson, S., Throup, J. P., Stewart, G. S. \& Williams, P. (1999). A hierarchical quorum-sensing system in Yersinia pseudotuberculosis is involved in the regulation of motility and clumping. Mol Microbiol 33, 1267-1277.

Belas, M. R. \& Colwell, R. R. (1982). Adsorption kinetics of laterally and polarly flagellated Vibrio. J Bacteriol 151, 15681580.

Christensen, B. B., Sternberg, C., Andersen, J. B., Palmer, R. J., Nielsen, A. T., Givskov, M. \& Molin, S. (1999). Molecular tools for the study of biofilm physiology. Methods Enzymol 310, 20-42.

Clark, J. D. \& Maaløe, O. (1967). DNA replication and the division cycle in Escherichia coli. J Mol Biol 23, 99-112.

Connell., T. D., Metzger, D. J., Lynch, J. \& Folster, J. P. (1998). Endochitinase is transported to the extracellular milieu by the eps-encoded general secretory pathway of Vibrio cholerae. J Bacteriol 180, 5591-5600.

Costerton, J. W., Lewandowski, Z., Caldwell, D. E., Korber, D. R. \& Lappin-Scott, H. M. (1995). Microbial biofilms. Annu Rev Microbiol 49, 711-745.

Costerton, J. W., Stewart, P. S. \& Greenberg, E. P. (1999). Bacterial biofilms: a common cause of persistent infections. Science 284, 1318-1322.

Danese, P. N., Pratt, L. A., Dove, S. L. \& Kolter, R. (2000). The outer membrane protein, antigen 43 , mediates cell-to-cell interactions within Escherichia coli biofilms. Mol Microbiol 37, 424-432.

Darling, P., Chan, M., Cox, A. D. \& Sokol, P. A. (1998). Siderophore production by cystic fibrosis isolates of Burkholderia cepacia. Infect Immun 66, 874-877.

Davey, M. E. \& O'Toole, G. A. (2000). Microbial biofilms: from ecology to molecular genetics. Microbiol Mol Biol Rev 64, 847-867.

Davies, D. G., Parsek, M. R., Pearson, J. P., Iglewski, B. H., Costerton, J. W. \& Greenberg, E. P. (1998). The involvement of cell-to-cell signals in the development of a bacterial biofilm. Science 280, 295-298.

Eberl, L., Winson, M. K., Sternberg, C. \& 7 other authors (1996). Involvement of $\mathrm{N}$-acyl-L-homoserine lactone autoinducers in controlling the multicellular behaviour of Serratia liquefaciens. Mol Microbiol 20, 127-136.

Eberl, L., Molin, S. \& Givskov, M. (1999). Surface motility of Serratia liquefaciens MG1. J Bacteriol 181, 1703-1712.

Geisenberger, O., Givskov, M., Riedel, K., Høiby, N., Tümmler, B.
\& Eberl, L. (2000). Production of $N$-acyl-L-homoserine lactones by $P$. aeruginosa isolates from chronic lung infections associated with cystic fibrosis. FEMS Microbiol Lett 184, 273-278.

Gessner, A. R. \& Mortensen, J. E. (1990). Pathogenic factors of Pseudomonas cepacia isolates from patients with cystic fibrosis. $J$ Med Microbiol 33, 115-120.

Glessner, A., Smith, R. S., Iglewski, B. H. \& Robinson, J. B. (1999). Roles of Pseudomonas aeruginosa las and $r h l$ quorum-sensing systems in control of twitching motility. J Bacteriol 181, 1623-1629.

Gotschlich, A., Huber, B., Geisenberger, O. \& 11 other authors (2001). Synthesis of multiple $N$-acyl-homoserine lactones is widespread among the members of the Burkholderia cepacia complex. Syst Appl Microbiol 24, 1-14.

Govan, J. R. W. \& Deretic, V. (1996). Microbial pathogenesis in cystic fibrosis: mucoid Pseudomonas aeruginosa and Burkholderia cepacia. Microbiol Rev 60, 539-574.

Harshey, R. M. (1994). Bees aren't the only ones: swarming in gram-negative bacteria. Mol Microbiol 13, 389-394.

Herrero, M., de Lorenzo, V. \& Timmis, K. N. (1990). Transposon vectors containing non-antibiotic resistance selection markers for cloning and stable chromosomal insertion of foreign genes in gram-negative bacteria. J Bacteriol 172, 6557-6567.

Heydorn, A., Nielsen, A. T., Hentzer, M., Sternberg, C., Givskov, M., Ersbøll, B. K. \& Molin, S. (2000). Quantification of biofilm structures by the novel computer program comstat. Microbiology 146, 2395-2407.

Hoang, T. T., Karkhoff-Schweizer, R. R., Kutchma, A. J. \& Schweizer, H. P. (1998). A broad-host range Flp-FRT recombination system for site-specific excision of chromosomallylocated DNA sequences: application for isolation of unmarked Pseudomonas aeruginosa mutants. Gene 212, 77-86.

Hutchison, M. L., Poxton, I. R. \& Govan, J. R. W. (1998). Burkholderia cepacia produces a hemolysin that is capable of inducing apoptosis and degranulation of mammalian phagocytes. Infect Immun 66, 2033-2039.

Isles, A., Maclusky, I., Corey, M., Gold, R., Prober, C., Fleming, P. \& Levison, H. (1984). Pseudomonas cepacia infection in cystic fibrosis: an emerging problem. J Pediatr 104, 206-210.

de Kievit, T. R. \& Iglewski, B. H. (2000). Bacterial quorum sensing in pathogenic relationships. Infect Immun 68, 4839-4849.

Koch, C. \& Høiby, N. (1993). Pathogenesis of cystic fibrosis. Lancet 341, 1065-1069.

Köhler, T., Curty, L. K., Barja, F., van Delden, C. \& Pechère, J. C. (2000). Swarming of Pseudomonas aeruginosa is dependent on cell-to-cell signaling and requires flagella and pili. J Bacteriol 182, 5990-5996.

Kovach, M. E., Elzer, P. H., Hill, D. S., Robertson, G. T., Farris, M. A., Roop, R. M. \& Peterson, K. M. (1995). Four new derivatives of the broad-host-range cloning vector pBBR1MCS, carrying different antibiotic-resistance cassettes. Gene 166, 175-176.

Lam, J., Chan, R., Lam, K. \& Costerton, J. W. (1980). Production of mucoid microcolonies by Pseudomonas aeruginosa within infected lungs in cystic fibrosis. Infect Immun 28, 546-556.

Lewenza, S., Conway, B., Greenberg, E. P. \& Sokol, P. A. (1999). Quorum sensing in Burkholderia cepacia: identification of the LuxRI homologs CepRI. J Bacteriol 181, 748-765.

Lindum, P. W., Anthoni, U., Christophersen, C., Eberl, L., Molin, S. \& Givskov, M. (1998). N-Acyl-L-homoserine lactone autoinducers control production of an extracellular lipopeptide biosurfactant required for swarming motility of Serratia liquefaciens MG1. J Bacteriol 180, 6384-6388. 
Lonon, M. K., Woods, D. E. \& Straus, D. C. (1988). Production of lipase by clinical isolates of Pseudomonas cepacia. J Clin Microbiol 26, 979-984.

Loo, C. Y., Corliss, D. A. \& Ganeshkumar, N. (2000). Streptococcus gordonii biofilm formation: identification of genes that code for biofilm phenotypes. J Bacteriol 182, 1374-1382.

de Lorenzo, V. \& Timmis, K. N. (1994). Analysis and construction of stable phenotypes in Gram-negative bacteria with Tn5- and Tn10-derived mini-transposons. Methods Enzymol 235, 386-405.

McKenney, D., Brown, K. E. \& Allison, D. G. (1995). Influence of Pseudomonas aeruginosa exoproducts on virulence factor production in Burkholderia cepacia: evidence of interspecies communication. J Bacteriol 177, 6989-6992.

McKevitt, A. I., Bajaksouzian, S., Klinger, J. D. \& Woods, D. E. (1989). Purification and characterization of an extracellular protease from Pseudomonas cepacia. Infect Immun 57, 771-778.

McLean, R. J., Whiteley, M., Stickler, D. J. \& Fuqua, W. C. (1997). Evidence of autoinducer activity in naturally occurring biofilms. FEMS Microbiol Lett 154, 259-263.

Ochsner, U. A. \& Reiser, J. (1995). Autoinducer-mediated regulation of rhamnolipid biosurfactant synthesis in Pseudomonas aeruginosa. Proc Natl Acad Sci US A 92, 6424-6428.

Oka, A., Sugisaki, H. \& Takanami, M. (1981). Nucleotide sequence of the kanamycin resistance transposon Tn903. J Mol Biol 147, 217-226.

O'Toole, G. A. \& Kolter, R. (1998a). Initiation of biofilm formation in Pseudomonas fluorescens WCS365 proceeds via multiple, convergent signalling pathways: a genetic analysis. Mol Microbiol 28, 449-461.

O'Toole, G. A. \& Kolter, R. (1998b). Flagellar and twitching motility are necessary for Pseudomonas aeruginosa biofilm development. Mol Microbiol 30, 295-304.

O'Toole, G., Kaplan, H. B. \& Kolter, R. (2000). Biofilm formation as microbial development. Annu Rev Microbiol 54, 49-79.

Parsek, M. R. \& Greenberg, E. P. (2000). Acyl-homoserine lactone quorum sensing in Gram-negative bacteria: a signaling mechanism involved in associations with higher organisms. Proc Natl Acad Sci U S A 97, 8789-8793.

Pratt, L. A. \& Kolter, R. (1998). Genetic analysis of Escherichia coli biofilm formation: roles of flagella, motility, chemotaxis and type I pili. Mol Microbiol 30, 285-293.

Pratt, L. A. \& Kolter, R. (1999). Genetic analyses of bacterial biofilm formation. Curr Opin Microbiol 2, 598-603.

Römling, U., Fiedler, B., Bosshammer, J., Grothues, D., Greipel, J., von der Hardt, H. \& Tümmler, B. (1994). Epidemiology of chronic Pseudomonas aeruginosa infections in cystic fibrosis. J Infect Dis 170, 1616-1621.

Rosenberg, E. \& Ron, E. Z. (1999). High- and low-molecular mass microbial surfactants. Appl Microbiol Biotechnol 52, 154-162.

Saiman, L., Cacalano, G. \& Prince, A. (1990). Pseudomonas cepacia adherence to respiratory epithelial cells is enhanced by Pseudomonas aeruginosa. Infect Immun 58, 2578-2584.

Sambrook, J., Fritsch, E. F. \& Maniatis, T. (1989). Molecular Cloning: a Laboratory Manual, 2nd edn. Cold Spring Harbor, New York: Cold Spring Harbor Laboratory.

Sanchez-Romero, J. M., Diaz-Orejas, R. \& de Lorenzo, V. (1998).
Resistance to tellurite as a selection marker for genetic manipulations of Pseudomonas strains. Appl Environ Microbiol 64, 4040-4046.

Sanger, F., Nicklen, S. \& Coulson, A. R. (1977). DNA sequencing with chain-terminating inhibitors. Proc Natl Acad Sci US A 74, 5463-5467.

Schierholz, J. M., Beuth, J., König, D., Nürnberger, A. \& Pulverer, G. (1999). Antimicrobial substances and effects on sessile bacteria. Zentbl Bakteriol 289, 165-177.

Schwyn, B. \& Neilands, J. B. (1987). Universal chemical assay for the detection and determination of siderophores. Anal Biochem 160, 47-56.

Shaw, P. D., Ping, G., Daly, S. L., Cha, C., Cronan, J. E., Rinchart, K. L. \& Farrand, S. K. (1997). Detecting and characterizing $N$-acylhomoserine lactone signal molecules by thin-layer chromatography. Proc Natl Acad Sci US A 94, 6036-6041.

Singh, P. K., Schaefer, A. L., Parsek, M. R., Moninger, T. O., Welsh, M. J. \& Greenberg, E. P. (2000). Quorum-sensing signals indicate that cystic fibrosis lungs are infected with bacterial biofilms. Nature 407, 762-764.

Stickler, D. J., Morris, N. S., McLean, R. J. \& Fuqua, C. (1998). Biofilms on indwelling urethral catheters produce quorumsensing signal molecules in situ and in vitro. Appl Environ Microbiol 64, 3486-3490.

Tolker-Nielsen, T. \& Molin, S. (2000). Spatial organization of microbial biofilm communities. Microb Ecol 40, 75-84.

Tümmler, B. \& Kiewitz, C. (1999). Cystic fibrosis: an inherited susceptibility to bacterial respiratory infections. Mol Med Today 5, 351-358.

Watnick, P. I. \& Kolter, R. (1999). Steps in the development of a Vibrio cholerae El Tor biofilm. Mol Microbiol 34, 586-595.

Watnick, P. \& Kolter, R. (2000). Biofilm, city of microbes. J Bacteriol 182, 2675-2679.

Williams, P., Camara, M., Hardman, A. \& 7 other authors (2000). Quorum sensing and the population-dependent control of virulence. Philos Trans R Soc Lond B Biol Sci 355, 667-680.

Winson, M. K., Swift, S., Hill, P. J., Sims, C. M., Griesmayr, G., Bycroft, B. W., Williams, P. \& Stewart, G. S. A. B. (1998a). Engineering the luxCDABE genes from Photorhabdus luminescens to provide a bioluminescent reporter for constitutive and promoter probe plasmids and mini Tn5 constructs. FEMS Microbiol Lett 163, 193-202.

Winson, M. K., Swift, S., Fish, L., Throup, J. P., Jørgensen, F., Chhabra, S. R., Bycroft, B. W., Williams, P. \& Stewart, G. S. A. B. (1998b). Construction and analysis of $\operatorname{lu} x C D A B E$-based plasmid sensors for investigating $\mathrm{N}$-acyl homoserine lactone-mediated quorum sensing. FEMS Microbiol Lett 163, 185-192.

Xu, K. D., McFeter, G. A. \& Stewart, P. S. (2000). Biofilm resistance to antimicrobial agents. Microbiology 146, 547-549.

Yohalem, D. S. \& Lorbeer, J. W. (1994). Intraspecific metabolic diversity among strains of Burkholderia cepacia isolated from decayed onions, soils, and the clinical environment. Antonie Leeuwenhoek 65, 111-131.

Received 3 March 2001; revised 2 May 2001; accepted 8 May 2001. 